\title{
GENERALIZED DISPLACEMENTS AND MOMENTA FORMULATIONS OF AN ELECTROMECHANICAL PLUNGER
}

\author{
TAMÁS SzABÓ ${ }^{a}$ AND LÁSzló RÓNAI ${ }^{a}$ \\ ${ }^{a}$ Institute of Machine Tools and Mechatronics, University of Miskolc, \\ 3515 Miskolc-Egyetemváros, Hungary \\ szabo.tamas@uni-miskolc.hu, ronai.laszlo@uni-miskolc.hu
}

[Received: June 22, 2020; Accepted: August 14, 2020]

Dedicated to Professor Barna Szabó on the occasion of his 85th birthday

\begin{abstract}
This paper deals with four different derivations of the governing equations of a solenoid plunger with lumped-parameter. Energy-based modeling is employed with extended Hamilton's principle with independent generalized coordinates and generalized momenta in order to be applicable to composite Lagrange's equations. In the electromechanical models, displacements and charges are regarded to be generalized coordinates, mechanical momenta and flux linkages are the generalized momenta. The derived systems of differential equations are solved numerically with the Runge-Kutta method.
\end{abstract}

Mathematical Subject Classification: 37N30, 70H03

Keywords: plunger, lumped-paramter, energy-based modeling, composite Lagrange's equation

\section{INTRODUCTION}

A mechatronic problem can include mechanical, electrical, hydraulic, and thermal phenomena. The application of an energy-based approach is suitable for the uniform management of all of them [1]. The following domain-independent generalized variables are advisable to use in complex problems: generalized coordinates, generalized momenta, generalized potentials and generalized velocities. The time derivative of the generalized potentials are often called efforts, and the generalized velocities are named flows. In this context generalized power, generalized energy, generalized potential energy, generalized potential co-energy, generalized kinetic energy, and generalized kinetic co-energy can be defined [1]- 2].

A plunger $[3]$ is regarded as an electromechanical problem. A number of papers deal with modeling and simulation of hydraulic valves, which are also plungers [4]-[5]. In these papers, models of lumped-parameter are investigated using displacement, electrical current or flux linkage variables. Following that, in addition to the previously mentioned variables, mechanical momenta will also be used to derive the governing 
equations. To the best knowledge of the authors of this article such equations for plungers written by momenta have not been presented yet.

Next an electromechanical plunger is modeled, in which variables displacement of the spool, electrical charge are generalized coordinates, the mechanical momentum of the spool and the flux linkage are generalized momenta. The model of the plunger to be investigated can be composed via purely generalized coordinates, or purely generalized momenta, and also their mixed combinations.

This paper is organized as follows: Section 2 defines the structure of the plunger. Section 3 details four different formulations of the governing equations of the model. Section 4 represents results of the numerical computations. Concluding remarks are given in Section 5

\section{A LUmped-PARAMETER ElECTROMECHANiCAL PLUNGER}

The structure of the plunger is shown in Figure 1 , where $m$ is the mass of the spool, $k$ is the stiffness of the suspending spring, $r$ is the damping coefficient of the dashpot, $L(y)$ is the inductance of the coil of the electromagnet, $R$ is the resistor of the inductor, $U_{0}(t)$ is the voltage source of the circuit and $k_{c}$ and $r_{c}$ represent the resultant stiffness and the damping coefficients of the collision layer, respectively. The nominal gap of the plunger is denoted by $y_{0}$ in unexcited situation. When the switch is closed the coil produces a magnetic force, which attracts the spool downwards to the electromagnet. Collision takes place at $y=-y_{0}$.

At this point the viscoelastic layer is under compression, it means that two springs $k+k_{c}$ and two dashpots $r+r_{c}$ are connected parallel to the spool. The spring characteristic of the problem is shown in Figure 2, where $\tan \alpha=k$ and $\tan \alpha_{c}=k+k_{c}$. Due to this fact the investigated problem is nonlinear, also in its mechanical aspect. The penalty treatment of the mechanical contact problem is based on a similar elastic layer sometimes with nonlinear spring, as was recommended by Prof. B. Szabó 6].

It is assumed that the inductance depends on the displacement $y$ of the spool [2]:

$$
L(y)=\frac{L_{0}}{1+\left(y_{0}+y\right) / h},
$$

where $L_{0}$ is the inductance at zero gap, $h$ is a geometric size, which is shown in Figure 1. The Coulomb charge and the flux linkage are denoted by $q$, and $\lambda$, respectively. It is self evident that the potential is zero at the vicinity of the ground.

In the sequel, the mathematical models of the plunger will be formulated on an energy basis using the extended Hamilton's principle with independent generalized coordinates, generalized momenta and their combinations.

\section{Mathematical formulation of the Problem}

A mechatronic system usually contains energy conservative and non-conservative elements. To treat such system the extended Hamilton's principle is a suitable variational principle: 


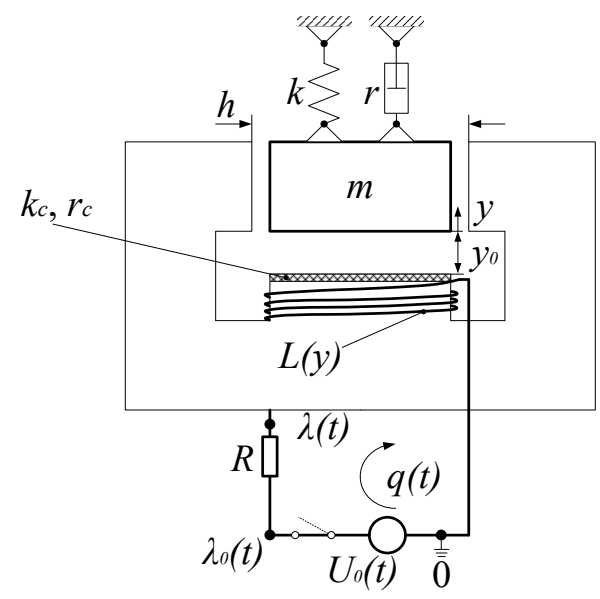

Figure 1. Schematic of the plunger

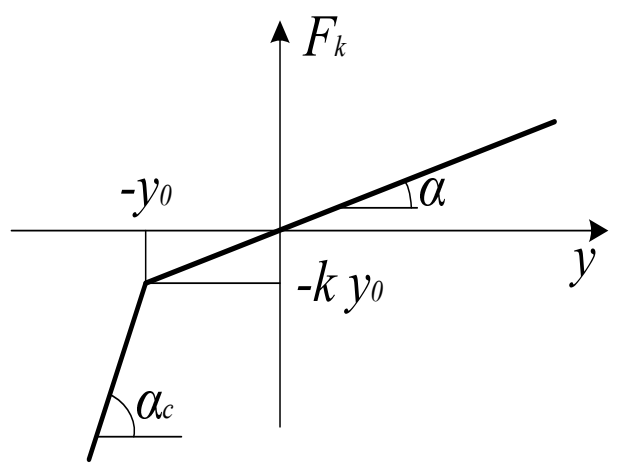

Figure 2. Spring characteristic of the electromechanical plunger

$$
\int_{t_{1}}^{t_{2}}\left(\delta \mathcal{L}+\overline{\delta W}_{n c}\right) d t=0, \quad \delta q_{k}=0 \quad \text { or } \quad \delta p_{k}=0, \quad k=1,2, \ldots, n ; \quad t=t_{1}, t_{2},
$$

where $\delta \mathcal{L}$ is the variation of the Lagrangian function containing energies and coenergies of the conservative elements, $\overline{\delta W}_{n c}$ is the virtual work associated to the non-conservative elements, $\delta q_{k}$ and $\delta p_{k}$ are the variation of the general coordinates and momenta of a system, respectively.

An electromechanical problem can be treated by four combinations of displacement $y$ and charge $q$ as generalized coordinates, and also mechanical momentum $p$ and flux linkage $\lambda$ as generalized momenta: 


$$
\begin{aligned}
& \mathcal{L}(y, q)=T^{*}(\dot{y})+W_{m}^{*}(\dot{q})-V_{p}(y)-W_{e}(q), \quad \overline{\delta W}_{n c}(y, q), \\
& \mathcal{L}(y, \lambda)=T^{*}(\dot{y})+W_{e}^{*}(\dot{\lambda})-V_{p}(y)-W_{m}(\lambda), \quad \overline{\delta W}_{n c}(y, \lambda), \\
& \mathcal{L}(p, q)=V_{p}^{*}(\dot{p})+W_{m}^{*}(\dot{q})-T(p)-W_{e}(q), \quad \overline{\delta W}_{n c}(p, q), \\
& \mathcal{L}(p, \lambda)=V_{p}^{*}(\dot{p})+W_{e}^{*}(\dot{\lambda})-T(p)-W_{m}(\lambda), \quad \overline{\delta W}_{n c}(p, \lambda),
\end{aligned}
$$

where $T^{*}(\dot{y})$ is the kinetic co-energy, $W_{m}^{*}(\dot{q})$ is the magnetic co-energy, $V_{p}(y)$ is the potential energy, $W_{m}(\lambda)$ is the magnetic energy, $V_{p}^{*}(p)$ is the potential co-energy, and $T(p)$ is the kinetic energy. Since the investigated system has no capacitor the electric energy $W_{e}(q)$ and co-energy $W_{e}^{*}(\dot{\lambda})$ are zero.

Virtual works in equations $3.2-(3.5)$ of the non-conservative elements are calculated by multiplication of the virtual change in generalized coordinates or generalized potentials and their energetically conjugated generalized forces or generalized velocities, respectively.

In order to apply the energy-based approach, it is noted that generally the admissibility requirements include kinematical constraints on the virtual displacements and the velocities, and Kirchhoff's current rule on the virtual charges and the currents. The admissibility requirements include the equation of Newton's second law on the virtual mechanical momenta and forces, and Kirchhoff's voltage rule on the virtual flux linkages and the voltages. Usually it is convenient to satisfy admissibility conditions on the virtual variations, i.e., to select a complete set of independent generalized coordinates [2].

Performing the variation of the Lagrangian function in equation (3.1), the Lagrange equation of the second kind can be obtained for independent generalized coordinates and momenta.

3.1. Displacement and charge formulation $(y, q)$. The most natural formulation of the plunger is using displacement and charge variables $3-7]$, since it is easy to satisfy the kinematical constraints and the Kirchhoff's current rule.

If the spool is not in contact with the collision layer, i.e., $\left(y+y_{0}\right)>0$ :

$$
\begin{gathered}
\mathcal{L}(y, \dot{y}, \dot{q})=\frac{1}{2} m \dot{y}^{2}+\frac{1}{2} L(y) \dot{q}^{2}-\frac{1}{2} k y^{2}, \\
\overline{\delta W}_{n c}=-r \dot{y} \delta y+U_{0}(t) \delta q-R \dot{q} \delta q,
\end{gathered}
$$

while in contact, i.e., $\left(y+y_{0}\right) \leq 0$ :

$$
\begin{gathered}
\mathcal{L}(y, \dot{y}, \dot{q})=\frac{1}{2} m \dot{y}^{2}+\frac{1}{2} L(y) \dot{q}^{2}-\frac{1}{2} k y^{2}-\frac{1}{2} k_{c}\left(y+y_{0}\right)^{2}, \\
\overline{\delta W}_{n c}=-\left(r+r_{c}\right) \dot{y} \delta y+U_{0}(t) \delta q-R \dot{q} \delta q,
\end{gathered}
$$

where $\delta y, \delta q$ are the variation of the displacement and the charge, respectively.

The governing equations are derived with the Lagrange equations: 


$$
\begin{aligned}
& \frac{d}{d t}\left(\frac{\partial \mathcal{L}}{\partial \dot{q}}\right)-\frac{\partial \mathcal{L}}{\partial q}=U_{0}(t)-R \dot{q}, \\
& L(y) \ddot{q}+R \dot{q}+L^{\prime}(y) \dot{y} \dot{q}=U_{0}(t),
\end{aligned}
$$

where $L^{\prime}(y)=\frac{d L(y)}{d y}$.

If the spool is not in contact with the collision layer, i.e., $\left(y+y_{0}\right)>0$ :

$$
\begin{aligned}
& \frac{d}{d t}\left(\frac{\partial \mathcal{L}}{\partial \dot{y}}\right)-\frac{\partial \mathcal{L}}{\partial y}=-r \dot{y}, \\
& m \ddot{y}+r \dot{y}+k y=L^{\prime}(y) \frac{\dot{q}^{2}}{2},
\end{aligned}
$$

while in contact $\left(y+y_{0}\right) \leq 0$ :

$$
\begin{gathered}
\frac{d}{d t}\left(\frac{\partial \mathcal{L}}{\partial \dot{y}}\right)-\frac{\partial \mathcal{L}}{\partial y}=-\left(r+r_{c}\right) \dot{y}, \\
m \ddot{y}+\left(r+r_{c}\right) \dot{y}+k y+k_{c}\left(y+y_{0}\right)=L^{\prime}(y) \frac{\dot{q}^{2}}{2} .
\end{gathered}
$$

The resulted ordinary differential equation equation (3.11) is the Kirchhoff's voltage rule, equations $(3.13)$ and $(3.15$ are Newton's second law.

3.2. Displacement and flux linkage formulation $(y, \lambda)$. The flux linkage is often used with the displacement to derive the equations [1, 2]. Instead of Kirchhoff's current rule, Kirchhoff's voltage rule should be satisfied for the flux linkages. The voltage source $U_{0}(t)$ can be expressed as the time derivative of a given function of the flux linkage $\dot{\lambda}_{0}(t)$. The selection of the flux linkage variable is based on Kirchhoff's voltage rule $U_{0}(t)=U_{R}+U_{L}=\left(\dot{\lambda}_{0}-\dot{\lambda}\right)_{R}+(\dot{\lambda}-0)_{L}$.

If the spool is not in contact, i.e., $\left(y+y_{0}\right)>0$ :

$$
\begin{gathered}
\mathcal{L}(y, \dot{y}, \lambda)=\frac{1}{2} m \dot{y}^{2}-\frac{1}{2} k y^{2}-\frac{\lambda^{2}}{2 L(y)}, \\
\overline{\delta W}_{n c}=-r \dot{y} \delta y-\frac{\dot{\lambda}-U_{0}(t)}{R} \delta \lambda,
\end{gathered}
$$

while in contact, i.e., $\left(y+y_{0}\right) \leq 0$ :

$$
\begin{gathered}
\mathcal{L}(y, \dot{y}, \lambda)=\frac{1}{2} m \dot{y}^{2}-\frac{1}{2} k y^{2}-\frac{1}{2} k_{c}\left(y+y_{0}\right)^{2}-\frac{\lambda^{2}}{2 L(y)}, \\
\overline{\delta W}_{n c}=-\left(r+r_{c}\right) \dot{y} \delta y-\frac{\dot{\lambda}-U_{0}(t)}{R} \delta \lambda,
\end{gathered}
$$

where $\delta \lambda$ is the variation of the flux linkage.

The governing equations are also derived with the Lagrange equations: 


$$
\begin{gathered}
\frac{d}{d t}\left(\frac{\partial \mathcal{L}}{\partial \dot{\lambda}}\right)-\frac{\partial \mathcal{L}}{\partial \lambda}=-\frac{\dot{\lambda}-U_{0}(t)}{R} \delta \lambda, \\
\frac{\dot{\lambda}}{R}+\frac{\lambda}{L(y)}=\frac{U_{0}(t)}{R} .
\end{gathered}
$$

If the spool is not in contact $\left(y+y_{0}\right)>0$ :

$$
\begin{gathered}
\frac{d}{d t}\left(\frac{\partial \mathcal{L}}{\partial \dot{y}}\right)-\frac{\partial \mathcal{L}}{\partial y}=-r \dot{y}, \\
m \ddot{y}+r \dot{y}+k y=\frac{1}{2} \lambda^{2} L(y)^{-2} L^{\prime}(y),
\end{gathered}
$$

while in contact $\left(y+y_{0}\right) \leq 0$ :

$$
\begin{gathered}
\frac{d}{d t}\left(\frac{\partial \mathcal{L}}{\partial \dot{y}}\right)-\frac{\partial \mathcal{L}}{\partial y}=-\left(r+r_{c}\right) \dot{y}, \\
m \ddot{y}+\left(r+r_{c}\right) \dot{y}+k y+k_{c}\left(y+y_{0}\right)=\frac{1}{2} \lambda^{2} L(y)^{-2} L^{\prime}(y) .
\end{gathered}
$$

The resulting differential equation (3.21) is the Kirchhoff's current rule, equations 3.23 and (3.25) are Newton's second law.

3.3. Mechanical momentum and charge formulation $(p, q)$. Mechanical momenta are applied less frequently compared to displacements in dynamical problems [1]. In order to satisfy the admissibility constraint on the mechanical momenta, Newton's second law should be considered:

$$
\dot{p}_{m}=\dot{p}_{k}+\dot{p}_{r}-\dot{p}_{M}
$$

where $\dot{p}_{m}$ is the time derivative of the linear momentum of the mass $m, \dot{p}_{k}$ is the spring force, $\dot{p}_{r}$ is the damping force, and $\dot{p}_{M}$ is the electromagnetic force.

The constraint equation for adequate mechanical momenta is obtained by taking the time integration of 3.26 :

$$
p_{m}=p_{k}+p_{r}-p_{M} .
$$

The Lagrangian function and the virtual work of the non-conservative elements are given as:

if the spool is not in contact, i.e., $\left(y+y_{0}\right)>0$ :

$$
\begin{gathered}
\mathcal{L}(p, \dot{p}, \dot{q})=\frac{\dot{p}_{k}^{2}}{2 k}+\frac{1}{2} L(y) \dot{q}^{2}-\frac{\left(p_{k}+p_{r}-p_{M}\right)^{2}}{2 m}, \\
\overline{\delta W}_{n c}=U_{0}(t) \delta q-R \dot{q} \delta q-\frac{\dot{p}_{r}}{r} \delta p_{r},
\end{gathered}
$$

while in contact, i.e., $\left(y+y_{0}\right) \leq 0$ : 


$$
\begin{gathered}
\mathcal{L}(p, \dot{p}, \dot{q})=\frac{\left(k y_{0}\right)^{2}}{2 k}+y_{0}\left(\dot{p}_{k}-k y_{0}\right)+\frac{\left(\dot{p}_{k}-k y_{0}\right)^{2}}{2\left(k+k_{c}\right)}+\frac{1}{2} L(y) \dot{q}^{2}-\frac{\left(p_{k}+p_{r}-p_{M}\right)^{2}}{2 m}, \\
\overline{\delta W}_{n c}=U_{0}(t) \delta q-R \dot{q} \delta q-\frac{\dot{p}_{r}}{r+r_{c}} \delta p_{r} .
\end{gathered}
$$

In equations (3.28)-(3.31) the displacement $y$ and the momenta $p_{m}, p_{M}$ are not independent variables; they can be given by two constitutive equations in which $\dot{q}, p_{k}$ and $p_{r}$ are the independent variables:

$$
\begin{gathered}
\dot{y}=\frac{\left(p_{k}+p_{r}-p_{M}\right)}{m}, \\
\dot{p}_{M}=-\frac{\partial W_{m}^{*}}{\partial y}=-\frac{1}{2} L^{\prime}(y) \dot{q}^{2} .
\end{gathered}
$$

The Lagrange equations are of the form

$$
\begin{gathered}
\frac{d}{d t}\left(\frac{\partial \mathcal{L}}{\partial \dot{q}}\right)-\frac{\partial \mathcal{L}}{\partial q}=U_{0}(t)-R \dot{q}, \\
L(y) \ddot{q}+R \dot{q}+L^{\prime}(y) \dot{y} \dot{q}=U_{0}(t .
\end{gathered}
$$

Substituting equation 3.32 into equation 3.35 yields:

$$
L(y) \ddot{q}+R \dot{q}+L^{\prime}(y) \frac{\left(p_{k}+p_{r}-p_{M}\right)}{m} \dot{q}=U_{0}(t) .
$$

If the spool is not in contact $\left(y+y_{0}\right)>0$ :

$$
\begin{gathered}
\frac{d}{d t}\left(\frac{\partial \mathcal{L}}{\partial \dot{p}_{k}}\right)-\frac{\partial \mathcal{L}}{\partial p_{k}}=0, \\
\frac{\ddot{p}_{k}}{k}+\frac{p_{k}+p_{r}-p_{M}}{m}=0, \\
\frac{d}{d t}\left(\frac{\partial \mathcal{L}}{\partial \dot{p}_{r}}\right)-\frac{\partial \mathcal{L}}{\partial p_{r}}=-\frac{\dot{p}_{r}}{r} \\
\frac{\dot{p}_{r}}{r}+\frac{p_{k}+p_{r}-p_{M}}{m}=0
\end{gathered}
$$

while in contact $\left(y+y_{0}\right) \leq 0$ :

$$
\begin{gathered}
\frac{d}{d t}\left(\frac{\partial \mathcal{L}}{\partial \dot{p}_{k}}\right)-\frac{\partial \mathcal{L}}{\partial p_{k}}=0, \\
\frac{\ddot{p}_{k}}{k+k_{c}}+\frac{p_{k}+p_{r}-p_{M}}{m}=0,
\end{gathered}
$$




$$
\begin{gathered}
\frac{d}{d t}\left(\frac{\partial \mathcal{L}}{\partial \dot{p}_{r}}\right)-\frac{\partial \mathcal{L}}{\partial p_{r}}=-\frac{\dot{p}_{r}}{r+r_{c}} \\
\frac{\dot{p}_{r}}{r+r_{c}}+\frac{p_{k}+p_{r}-p_{M}}{m}=0 .
\end{gathered}
$$

The resulted ordinary differential equation 3.36 is Kirchhoff's voltage rule, equations (3.38), 3.40), 3.42), and (3.44) are the kinematical connection equations.

3.4. Mechanical momentum and flux linkage formulation $(p, \lambda)$. This formulation is based on purely the generalized momenta, i.e., on the mechanical momenta and flux linkage. Here in addition to the admissibility constraints equations (3.26) and (3.27) valid for the mechanical momenta, Kirchhoff's voltage rule should also be satisfied for the flux linkage as in Subsection 3.2

The Lagrangian function and the virtual work of the non-conservative elements are given as:

if the spool is not in contact, i.e., $\left(y+y_{0}\right)>0$ :

$$
\begin{gathered}
\mathcal{L}(p, \dot{p}, \lambda)=\frac{\dot{p}_{k}^{2}}{2 k}-\frac{\left(p_{k}+p_{r}-p_{M}\right)^{2}}{2 m}-\frac{\lambda^{2}}{2 L(y)}, \\
\overline{\delta W}_{n c}=-\frac{\dot{\lambda}-U_{0}}{R} \delta \lambda-\frac{\dot{p}_{r}}{r} \delta p_{r}
\end{gathered}
$$

while in contact, i.e., $\left(y+y_{0}\right) \leq 0$ :

$$
\begin{gathered}
\mathcal{L}(p, \dot{p}, \lambda)=\frac{\left(k y_{0}\right)^{2}}{2 k}+y_{0}\left(\dot{p}_{k}-k y_{0}\right)+\frac{\left(\dot{p}_{k}-k y_{0}\right)^{2}}{2\left(k+k_{c}\right)}-\frac{\left(p_{k}+p_{r}-p_{M}\right)^{2}}{2 m}-\frac{\lambda^{2}}{2 L(y)}, \\
\overline{\delta W}_{n c}=-\frac{\dot{\lambda}-U_{0}}{R} \delta \lambda-\frac{\dot{p}_{r}}{r+r_{c}} \delta p_{r} .
\end{gathered}
$$

In equations 3.45-3.48 $y$ and $p_{M}$ are also not independent; they should satisfy the following constitutive equations:

$$
\begin{gathered}
\dot{y}=\frac{\left(p_{k}+p_{r}-p_{M}\right)}{m}, \\
\dot{p}_{M}=\frac{\partial W_{m}}{\partial y}=-\frac{1}{2} L^{\prime}(y) \lambda^{2} L(y)^{-2} .
\end{gathered}
$$

The Lagrange equations can be written as:

$$
\begin{gathered}
\frac{d}{d t}\left(\frac{\partial \mathcal{L}}{\partial \dot{\lambda}}\right)-\frac{\partial \mathcal{L}}{\partial \lambda}=-\frac{\dot{\lambda}-U_{0}}{R}, \\
\frac{\dot{\lambda}-U_{0}}{R}+\frac{\lambda}{L(y)}=0 .
\end{gathered}
$$


If the spool is not in contact $\left(y+y_{0}\right)>0$ :

$$
\begin{gathered}
\frac{d}{d t}\left(\frac{\partial \mathcal{L}}{\partial \dot{p}_{k}}\right)-\frac{\partial \mathcal{L}}{\partial p_{k}}=0, \\
\frac{\ddot{p}_{k}}{k}+\frac{p_{k}+p_{r}-p_{M}}{m}=0, \\
\frac{d}{d t}\left(\frac{\partial \mathcal{L}}{\partial \dot{p}_{r}}\right)-\frac{\partial \mathcal{L}}{\partial p_{r}}=-\frac{\dot{p}_{r}}{r}, \\
\frac{\dot{p}_{r}}{r}+\frac{p_{k}+p_{r}-p_{M}}{m}=0,
\end{gathered}
$$

while in contact $\left(y+y_{0}\right) \leq 0$ :

$$
\begin{gathered}
\frac{d}{d t}\left(\frac{\partial \mathcal{L}}{\partial \dot{p}_{k}}\right)-\frac{\partial \mathcal{L}}{\partial p_{k}}=0 \\
\frac{\ddot{p}_{k}}{k+k_{c}}+\frac{p_{k}+p_{r}-p_{M}}{m}=0, \\
\frac{d}{d t}\left(\frac{\partial \mathcal{L}}{\partial \dot{p}_{r}}\right)-\frac{\partial \mathcal{L}}{\partial p_{r}}=-\frac{\dot{p}_{r}}{r+r_{c}}, \\
\frac{\dot{p}_{r}}{r+r_{c}}+\frac{p_{k}+p_{r}-p_{M}}{m}=0
\end{gathered}
$$

The resulting differential equation 3.52 is Kirchhoff's current rule, Equations (3.54), 3.56 3.58), 3.60 are the kinematical connection equations.

\section{NumericAl ANALYSIS}

A special purpose program has been developed using Scilab software for numerical solutions of the four set of systems of differential equations. The Runge-Kutta numerical method has been used in order to get the results. The problem shown in Figure 1 has been analyzed with the following parameters: $L_{0}=1.5 \mathrm{mH}, y_{0}=2.5 \mathrm{~mm}$, $h=10 \mathrm{~mm}, R=2.4 \Omega, U_{0}=12 \mathrm{~V}, \mathrm{~m}=10 \mathrm{~g}, \mathrm{k}=500 \mathrm{~N} / \mathrm{m}, \mathrm{r}=1.5 \mathrm{Ns} / \mathrm{m}$, $k_{c}=5 \cdot 10^{6} \mathrm{~N} / \mathrm{m}$, and $r_{c}=1500 \mathrm{Ns} / \mathrm{m}$.

The displacement coordinate $y$ versus time $t$ has been computed with four different formulations and equal results have been obtained, which are shown in Figure 3. Equal diagrams of current $\dot{q}$ versus time $t$ have been computed with (displacement - charge), and (momentum - charge) formulations (see Figure 4). Equal curves of flux linkage $\lambda$ versus time $t$ have been obtained by (displacement - flux linkage), and (momentum flux linkage) formulations (see Figure 5). Equal curves have been determined for the momentum $p_{m}$ of the spool versus time $t$ by (momentum - charge) and (momentum - flux linkage) formulations (see Figure 6).

Multiple collisions of the spool with decreasing amplitude of rebounds can be recognized in Figure 3 and Figure6, then the spool comes to a standstill. There are also 
correlations between the curves of the current, the flux linkage and the motion of the spool (see Figure 5 and Figure 6).

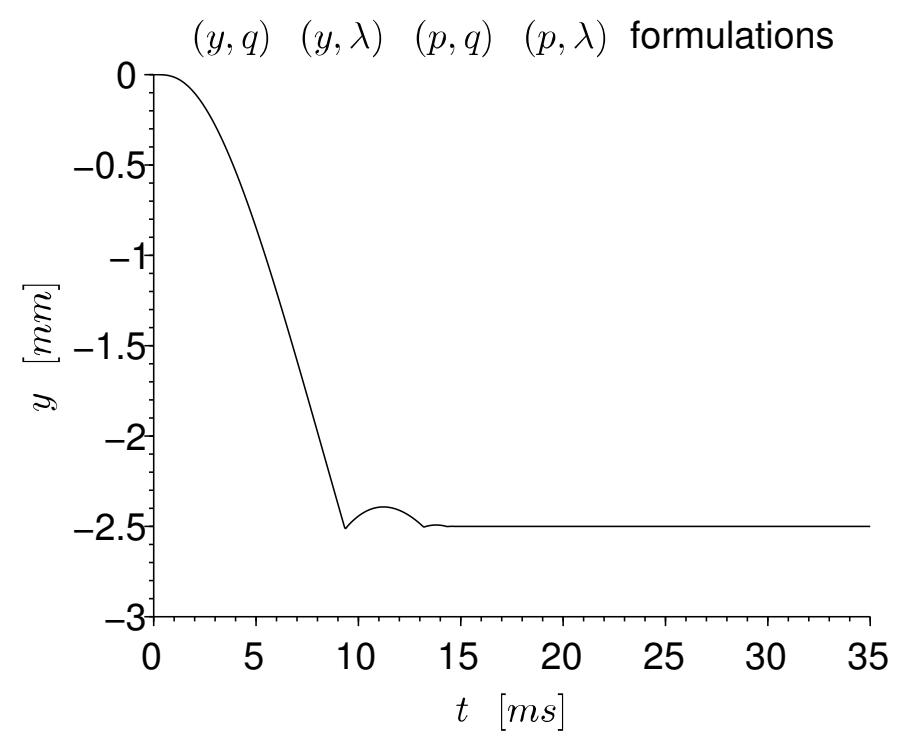

Figure 3. Displacement of the spool

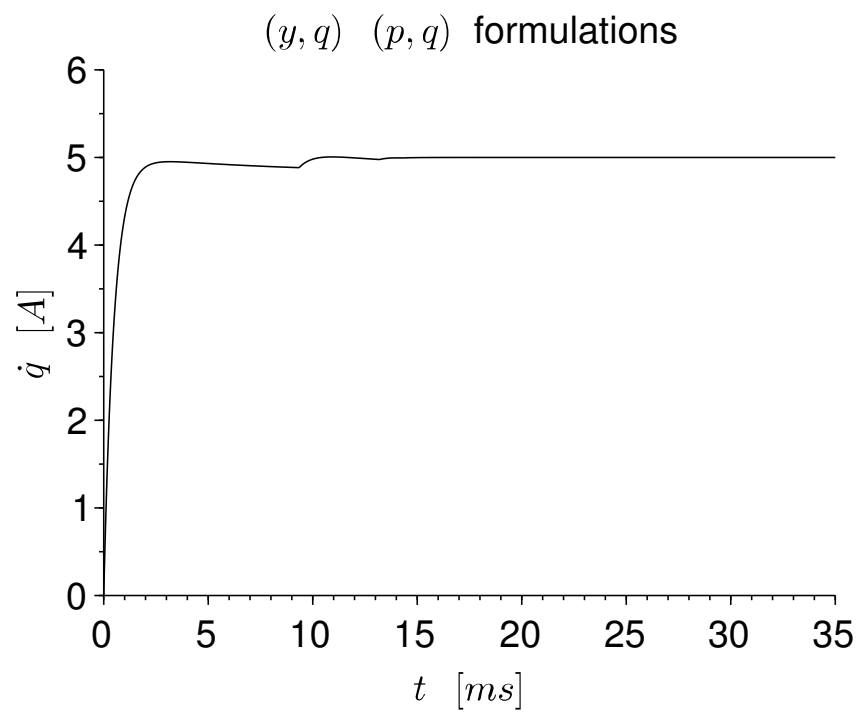

Figure 4. Current of the coil 


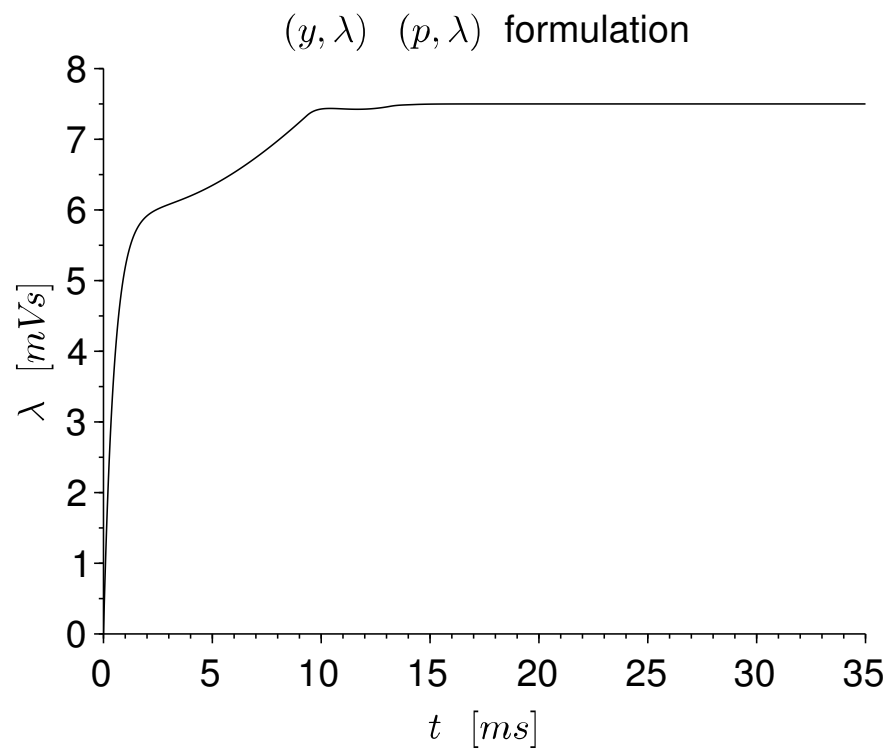

Figure 5. Flux linkage of the electromagnet

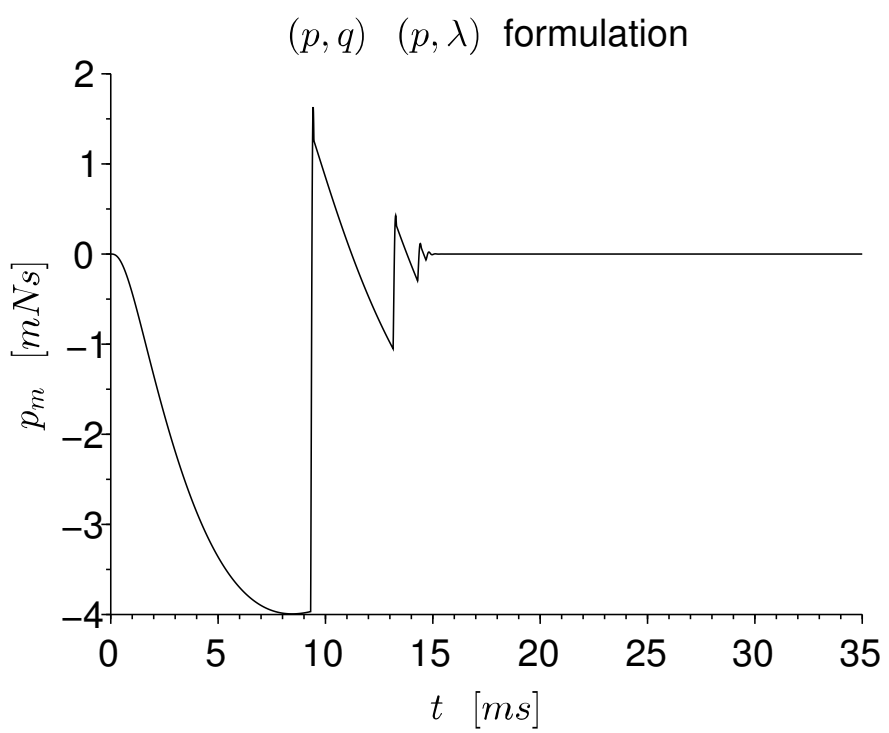

Figure 6. Momentum of the spool 


\section{Conclusions}

Four equivalent formulations have been presented to model an electromechanical plunger using generalized coordinates and generalized momenta. The system of equations obtained by two formulations containing mechanical momenta can be regarded as novel results.

Numerical computations confirmed the equivalence of the four formulations. When a displacement coordinate is used in the formulations together with charge or flux linkage, three differential equations are obtained in both cases. However, seven differential equations are derived by mechanical momentum with charge or flux linkage formulation. Usually it is more convenient to satisfy the constraints for the generalized coordinates than their counterparts of momenta. Thus a formulation based on generalized coordinates is more popular compared to the generalized momenta formulations.

Acknowledgement. The described article was carried out as part of the EFOP-3.6.1-162016-00011 "Younger and Renewing University - Innovative Knowledge City - institutional development of the University of Miskolc aiming at intelligent specialisation" project implemented in the framework of the Szechenyi 2020 program. The realization of this project is supported by the European Union, co-financed by the European Social Fund.

\section{REFERENCES}

1. P. E. Wellstead. Introduction to Physical System Modelling. Academic Press Ltd., London, 1979.

2. A. Preumont. Mechatronics - Dynamics of Electromechanical and Piezoelectric Systems. Springer-Verlag, Berlin, Heidelberg, 2006. DOI: 10.1007/1-4020-46960 .

3. A. Demarchi, L. Farçoni, A. Pinto, R. Lang, R. Romero, and I. Silva. "Modelling a Solenoid's Valve Movement." RoboCup 2017: Robot World Cup XXI. Ed. by H. Akiyama, O. Obst, C. Sammut, and F. Tonidandel. Cham: Springer International Publishing, 2018, pp. 290-301. DOI: 10.1007/978-3-030-00308-1_24.

4. H. Tian and Y. Zhao. "Coil inductance model based solenoid on-off valve spool displacement sensing via laser calibration." Sensors, 18(12), (2018), pp. 1-14. DOI: $10.3390 / \mathrm{s} 18124492$.

5. A. E. Balau and C. Lazar. "State-space model of an electro-hydraulic actuated wet clutch." IFAC Proceedings Volumes, 6th IFAC Symposium Advances in Automotive Control, Munich, Germany. IFAC, 2010, pp. 506-511. DOI: 10.3182/ 20100712-3-DE-2013.00039

6. Y. Volpert, T. Szabó, I. Páczelt, and B. Szabó. "Application of the space enrichment method to problems of mechanical contact." Finite Elements in Analysis and Design, 24(3), (1997), pp. 157-170. DOI: 10.1016/S0168-874X (96) 00047-9.

7. A. C. Yudell and J. D. Van de Ven. "Predicting solenoid valve spool displacement through current analysis." International Journal of Fluid Power, 16(3), (2015), pp. 133-140. DOI: 10.1080/14399776.2015.1068549. 